\title{
Regenerative Engineering: Approaches to Limb Regeneration and Other Grand Challenges
}

\author{
Cato T. Laurencin ${ }^{1,2,3,4,5,6} \cdot$ Lakshmi S. Nair ${ }^{1,2,3,4,5}$ \\ Received: 18 November 2015 /Revised: 20 November 2015 / Accepted: 20 November 2015 /Published online: 4 December 2015 \\ (C) The Regenerative Engineering Society 2015
}

\begin{abstract}
The clinical grand challenge to regenerate complex tissue and organ systems calls for a paradigm shift that requires a transdisciplinary approach. The field of regenerative engineering puts forward a convergence approach to create a regenerative toolbox to move beyond individual tissue repair to the regeneration of complex tissues and organ systems. Here, we discuss the regenerative tool box currently under development to address grand opportunities in complex tissue/organ regeneration.
\end{abstract}

\section{Lay Summary}

Regenerating damaged tissues rather than repairing or replacing is the holy-grail to address the clinical challenge of organ damage. Unlike advanced prosthetics, the field of "Regenerative Engineering" aims to completely regenerate complex tissues and organ systems such as a knee or a whole limb.

Cato T. Laurencin

laurencin@uchc.edu

1 Department of Orthopaedic Surgery, University of Connecticut Health Center, Farmington, CT 06030, USA

2 Institute for Regenerative Engineering, University of Connecticut Health Center, Farmington, CT 06030, USA

3 Raymond and Beverly Sackler Center for Biomedical, Biological, Physical and Engineering Sciences, University of Connecticut Health Center, Farmington, CT 06030, USA

4 Department of Biomedical Engineering, University of Connecticut, Storrs, CT 06269, USA

5 Department of Materials Science and Engineering, University of Connecticut, Storrs, CT 06269, USA

6 Department of Chemical and Biomolecular Engineering, University of Connecticut, Storrs, CT 06269, USA
Keywords Regenerative engineering · Convergence · Advanced biomaterials · Developmental biology $\cdot$ Stem cells . Physical forces

The regenerative capability of the adult human body is surprisingly very high. Blood is a paramount example that is continuously regenerated throughout a person's life. Several other tissues such as bone have the capability to regenerate to a certain extent upon injury. Multipotent reserve stem/progenitor cell populations have been identified throughout the adult human body and have the capability to assist the regenerative process [1]. Despite their tremendous potential, the majority of adult human tissues is incapable of true regeneration and instead follows a repair process leading to scar tissue formation that lacks necessary functional and biological properties.

Tissue loss due to injury or disease and the subsequent functional impairment continues to be one of the significant challenges of modern medicine. Use of prosthetics to replace the damaged tissue is one of the currently used strategies to address this issue. The past 50 years or so have seen dramatic advances in the prosthetic technology; however, the field still suffers from serious shortcomings such as functional limitations, mechanical incompatibility, poor host tissue integration, and long-term biocompatibility issues [2]. Another well sought after approach to address the clinical challenge of restoring the function of damaged/injured organ or tissue is the use of biological grafts. Current biological grafts include tissue transplanted within an individual from a donor site to the injury site commonly referred to as autografts or tissue/organ isolated from another individual or cadaver, which is referred to as allografts. Among the biological grafts, autografts are considered the gold standard due to their excellent biocompatibility and healing potential. The limited availability of autografts and biological challenges with allografts such as need for immunosuppression have led to 
the emergence of alternative clinical interventions focused on regenerating the damaged tissues rather than repairing or replacing the tissues [3]. The field of tissue engineering, which can be defined as the application of biological, chemical, and engineering principles toward the repair, restoration, and regeneration of tissues using biomaterials, cells, and factors alone or in combination, evolved out of this necessity [4]. The triad combination strategy put forward by the tissue engineering approach led to several proof-of-concept technologies and demonstrated the feasibility to repair or regenerate individual tissues [5]. In spite of the significant advances in tissue engineering in the past 25 years, the number of tissue-engineered products that are helping people clinically are relatively low.

Developing innovative strategies to regenerate complex tissues and organ systems are key to move to the next level to create translational technologies that can address the clinical grand challenges. For example, from a musculoskeletal perspective, even though we have the capability to regenerate small segments of bone and tissues such as ligament, tendon, and cartilage, the grand clinical challenge lies in regenerating a functional knee or whole limb [6] http://www. connecticutmag.com/Blog/Health-Wellness/October-2015/ UConn-Professor-Announces-Goal-to-Regrow-HumanLimb-in-15-Years/. To address these clinical grand challenges, a paradigm shift is required. This needs a transdisciplinary use of cutting edge technologies in biology, material sciences, physical, chemical, and engineering sciences, combined with our ability to harness the body's innate capacity to guide growth and development. Engaging clinicians early on is important. These efforts have led to the emergence of a field termed as "Regenerative Engineering" [7]. Regenerative Engineering has been defined as the Convergence of Advanced Materials Sciences, Stem Cell Sciences, Physics, Developmental Biology, and Clinical Translation for the regeneration of complex tissues and organ systems. The tremendous advances we have achieved in these distinct areas have provided a toolbox that presents the scientists with exciting possibilities to usher the field of regenerative engineering forward to develop highly translational technologies. The excitement in this field lies in the potential of convergence - the transdisciplinary use of technologies and science - in moving the field forward toward new solutions for regeneration in humans. For instance, we now have a deeper understanding of adult and embryonic stem cells as well as the capability to obtain adult patient-derived stem cells with tremendous regenerative ability. Similarly, the field of biomaterials science has significantly advanced from the level of biodegradable polymers and ceramics to custom-designed biomimetic inductive biomaterials with carefully modulated physical, mechanical, and biological properties to enhance the natural regenerative process of the body [8-10]. The emergence of micro- and nanotechnologies fueled the developments in this area by lending new methodologies to create three-dimensional biomimetic scaffolds [11, 12]. The studies in this area so far enabled us to understand the cellular sensitivity toward the biological environment and therefore the potential to modulate the cellular functions using advanced biomaterials and biomimetic scaffolds. This along with the growing evidence of achieving better functional outcome in larger animals using a biomaterial approach compared to smaller animals mirrors the translational potential of inductive biomaterials.

Using top-down and bottom-up approaches, regenerative engineering could make complex tissue regeneration possible, conceivably addressing a number of grand challenges, such as limb regeneration. The bottom-up approach is largely cellular and is fueled by the tremendous advances we are having in the field of cell/molecular and developmental biology. Many lower amphibians have the remarkable ability to regenerate complex tissue and organ systems such as amputated limbs by the self-assembly of the proliferating and differentiating cells in blastema. This highly orchestrated biological process unfolds through a tightly controlled spatial and temporal expression of various morphogenetic cues. A deeper understanding of the regenerative process occurring in the amphibians has the potential to inform us to design novel translational strategies that can enhance the regenerative capabilities of human [13]. The top-down approaches are built on the integration of advanced material science and engineering, cells with high regenerating ability, physical forces that play a subtle but crucial part in the process of regeneration, modulating the immune system, as well as ways to design strategies to identify and present biologically active effector molecules and inducerons (small molecule inducers of cell differentiation) as the complexity of multi-tissues and organ systems requires input from each individual discipline. In short, the design of systems for regeneration must take place with a holistic and comprehensive approach, understanding the contributions of cells, biological factors, scaffolds, physical forces, and morphogenesis. We believe that the new journal "Regenerative Engineering and Translational Medicine" will serve as a platform to discuss the convergence approach put forward by the field of regenerative engineering. To move the field beyond individual tissue repair to the regeneration of complex tissues and organ systems is the goal of this new field, and its journal. 
Acknowledgments This work was supported by the NIH Director's Pioneer Award, 1DP1AR068147.

\section{References}

1. Murphy MB, Monvivais K, Caplan AI. Mesenchymal stem cells: environmentally responsive therapeutics for regenerative medicine. Exp Mol Med. 2013;45:e54.

2. Talmo CT, Aghazadeh M, Bono JV. Perioperative complications following total joint replacement. Clin Geriatr Med. 2012;28:47187.

3. Khademhossseini A, Vacanti JP, Langer R. Progress in tissue engineering. Sci Am. 2009;300:64-71.

4. Laurencin CT, Ambrosio AA, Bordern MD, Cooper JA. Annual Review of Biomedical Engineering. Yarmush MI (ed), Annual Reviews, Palo Alto . pp. 19-46 (1999)

5. Samadikuchaksaraei A. Scientific and industrial status of tissue engineering. Afr J Biotech. 2007;6:2897-909.

6. Reichert WM, Ratner BD, Anderson J, Coury A, Hoffman AS, Laurencin CT, et al. 2010 panel on the biomaterials grand challenges. J Biomed Mater Res A. 2011;96:275.
7. Laurencin CT, Khan Y. Regenerative engineering. Sci Transl Med. 4, 160ed9 (2012)

8. Cushnie EK, Ulery BD, Nelson SJ, Deng M, Sethuraman S, Doty $\mathrm{SB}$, et al. Simple signaling molecules for inductive bone regenerative engineering. PLoS One. 2014;9(7), e101627. doi:10.1371/ journal.pone.0101627. eCollection (2014).

9. Lo KW, Kan HM, Gagnon KA, Laurencin CT. One-day treatment of small molecule 8-bromo-cyclic AMP analogue induces cellbased VEGF production for in vitro angiogenesis and osteoblastic differentiation. J Tissue Eng Regen Med. 2013. doi:10.1002/term. 1839.

10. Cooper Jr JA, Sahota JS, Gorum 2nd WJ, Carter J, Doty SB, Laurencin CT. Biomimetic tissue-engineered anterior cruciate ligament replacement. Proc Natl Acad Sci U S A. 2007;104:3049-54.

11. Lv Q, Deng M, Ulery BD, Nair LS, Laurencin CT. Nano-ceramic composite scaffolds for bioreactor-based bone engineering. Clin Orthop Relat Res. 2013;471:2422-33.

12. Li WJ, Laurencin CT, Caterson EJ, Tuan RS, Ko FK. Electrospun nanofibrous structure: a novel scaffold for tissue engineering. J Biomed Mater Res. 2002;60:613-21.

13. Gardiner DM, Bryant S, Muneoka K. Engineering Limb Regeneration: Lessons from animals that can regenerate. In. Regenerative Engineering, Laurencin CT \& Khan Y (Eds), CRC Press, Taylor \& Francis (2013). 